The Nepal Math. Sc. Report

Vol. 34, No. 1 and 2, 2016

\title{
A REVIEW ON THE STRUCTURE AND PROPERTIES OF THE ESCAPING SET OF TRANSCENDENTAL ENTIRE FUNCTIONS
}

\author{
BISHNU HARI SUBEDI AND AJAYA SINGH \\ Central Department of Mathematics, Tribhuvan University, \\ Kathmandu, Nepal
}

\begin{abstract}
For a transcendental entire function $f$, we study the structure and properties of the escaping set $I(f)$ which consists of points whose iterates under $f$ escape to infinity. We concentrate on Eremenko's conjecture and we review some attempts of its proofs. A significant amount of progress in Eremenko's conjecture has been made possible via fast escaping set $A(f)$ which consists points that escape to infinity as fast as possible. This set can be written as union of closed sets, called levels of $A(f)$. We review classes of functions for which $A(f)$ and each of its levels has the structure of infinite spider's web. In general, we study classes of entire functions for which the escaping set $I(f)$ is a spider's web. Spider's web is a recently investigated structure of $I(f)$ that gives new results in the direction of Eremenko's conjecture.
\end{abstract}

Key Words: Escaping set, Eremenko's conjecture, Fast escaping set, Spider's web, Regularity condition etc.

AMS (MOS) Subject Classification. 37F10, 30D05.

\section{INTRODUCTION}

The subject complex dynamics formally originated by the independent work of Fatou [20, 21] and Julia [25] during 1917-1926. All these early papers of Fatou and Julia considered the iteration of rational functions. In this article, we consider only the iteration of transcendental entire function (TEF) which was initiated by Fatou [20] in 1926 and developed much more in the work of Baker $[2,3,4,5,6]$. Later, solid body of knowledge in transcendental iteration theory has developed in the work of Eremenko [15], Eremenko and Lyubich [16, 17, 18, 19], Bergweiler [7, 8, 9, 10, 11, 12, 13], Rippon and Stallard [37, 38, 39, 40, 41, 42, 44], Schleicher [48, 49, 50], Rempe [34, 35, 36], Sixsmith $[51,52,53,54,55]$ and Osborne $[28,29,30,31,32,33]$.

We denote the complex plane by $\mathbb{C}$ and set of integers greater than zero by $\mathbb{N}$. We assume the function $f: \mathbb{C} \rightarrow \mathbb{C}$ is transcendental entire function (TEF) unless otherwise stated. For any $n \in \mathbb{N}, f^{n}$ always denotes the nth iterates of $f$. The order $\rho(f)$ and lower order $\lambda(f)$ of TEF $f$ are defined respectively by

$$
\rho(f)=\limsup _{r \rightarrow \infty} \frac{\log \log M(r, f)}{\log r} \text { and } \lambda(f)=\liminf _{r \rightarrow \infty} \frac{\log \log m(r, f)}{\log r} .
$$

Received March 2, 2016 
where $M(r, f)=\max _{|z|=r}|f(z)|, r>0$ and $m(r, f)=\min _{|z|=r}|f(z)|, r>0$ denote respectively the maximum and minimum modulus of the function $f$. We will see in section 3 that these terms are important in the study of functions for which $A_{R}(f)$ is a spider's web.

A family $\mathcal{F}=\left\{f^{n}: n \in \mathbb{N}\right\}$ of the iterates of TEF $f$ forms a normal family if every sequence $\left(f^{n}\right)_{n \in \mathbb{N}}$ of functions contains a subsequence which converges uniformly to a limit $f \neq \infty$ or converges to $\infty$ on every compact subset $\mathrm{D}$ of $\mathbb{C}$. The Fatou set of $f$ denoted by $F(f)$ is the set of point's $z \in \mathbb{C}$ such that sequence $\left(f^{n}\right)_{n \in \mathbb{N}}$ forms a normal family in some neighborhood of $z$ in the sense of Montel. A connected component of the Fatou set $F(f)$ is called Fatou component. The complement of Fatou set is called Julia set and it is denoted by $J(f)$. The basic properties and structures of these sets can be found in [7, 14, 24, 26, 27].

For a TEF $f$, if $f^{\prime}(z)=0$, we say $z$ is a critical point and $w=f(z)$ is a critical value. For a TEF $f$, a curve $\Gamma:[0, \infty) \rightarrow \mathbb{C}$ is an asymptotic curve with asymptotic value $\alpha$ if $\Gamma(t) \rightarrow \infty$ and $f(\Gamma(t)) \rightarrow \alpha$ as $t \rightarrow \infty \forall t \in[0, \infty)$. The set $S V(f)=\overline{(C V(f) \cup A V(f))}$ is called set of singular values, where $C V(f)$ and $A V(f)$ respectively denote the set of critical values and asymptotic values. Note that the set $S V(f)$ coincides with the set of singularities of the inverse function $f^{-1}$ of $f$, and so this set is also denoted by $\operatorname{Sing}\left(f^{-1}\right)$. If $S V(f)$ has only finitely many elements, then $f$ is said to be of finite type. If $S V(f)$ is a bounded set, then $f$ is said to be of bounded type. The sets $\mathcal{S}=\{f: f$ is of finite type $\}$ and $\mathcal{B}=\{f: f$ is of bounded type $\}$ are respectively called Speiser class and EremenkoLyubich class. Note that $\rho(f) \geq \frac{1}{2}$ for any bounded type transcendental map and $\rho(f) \geq 1$ for any finite type map. The class $\mathcal{B}$ introduced in complex dynamics by Eremenko and Lyubich [16]. The most important result of this paper[16] is $F(f) \cap I(f)=\emptyset$ if $f \in \mathcal{B}$. The most familiar functions in this class are the functions in the exponential family $\{f: f(z)=$ $\lambda \exp (z), \lambda \neq 0\}$ and the functions in the cosine family $\{f: f(z)=\cos (\alpha z+\beta), \alpha \neq 0\}$

\section{Escaping Set and Eremenko's Conjecture}

In recent years, much interest and more effort have been devoted to understanding the structure and properties of the escaping set $I(f)$ of $f$ which is defined as follows:

Definition 2.1 (Escaping Set). : For a TEF $f$, the set of the form

$$
I(f)=\left\{z: f^{n}(z) \rightarrow \infty \text { as } n \rightarrow \infty\right\}
$$

is called escaping set.

For a TEF $f$, the escaping set $I(f)$ was first studied by A. Eremenko [15]. The fundamental properties of the escaping set $I(f)$ are as follows.

Theorem 2.1. For a TEF $f$, the following statements are hold.

(1) $I(f)=I\left(f^{n}\right)$ for $n \geq 2$.

(2) $I(f)$ is completely invariant.

(3) $I(f) \neq \emptyset$.

(4) $J(f) \cap I(f) \neq \emptyset$.

(5) $J(f)=\partial I(f)$. 
(6) $\overline{I(f)}$ has no bounded components.

The first two statements (1) and (2) of this theorem 2.1 follows from the definition of $I(f)$ and the rest (3), (4), (5) and (6) are proved in [15]. If $U$ is a Fatou component such that $U \cap I(f) \neq \emptyset$, then by normality $U \subset I(f)$. We say that such a Fatou component $U$ is escaping Fatou component. However, the boundary of such escaping Fatou component may not be in $I(f)$. For example, the function $f(z)=e^{-z}+z+1$ has escaping Fatou component but its boundary contains periodic points which are not in $I(f)$. On the basis of statement (6) of above theorem 2.1 and Ninty years old Fatou's original question [20] initiating from the functions such as $f(z)=e^{-z}+z+1$ and $f_{\lambda}=\lambda \sin z$ concerning whether there are infinitely many curves $\gamma_{k}, k \in \mathbb{N}$ such that $z \in \gamma_{k}, f^{n}(z) \rightarrow \infty$ as $n \rightarrow \infty$, Eremenko made the following conjectures in more precise form in [15].

Conjecture 2.1 (Normal (weak) version). Each component of $I(f)$ is unbounded.

Conjecture 2.2 (Strong version). Each escaping point can be connected to infinity along a unique curve within $I(f)$.

2.1. Attempt of Proving Normal Version of Eremenko's Conjecture. This conjecture in normal form in general case has been proved by using the fast escaping set $A(f)$, which consists of points whose iterates tends to infinity as fast as possible. This set is introduced first time by Bergweiler and Hinkkanen [13] and now plays a key role in transcendental dynamics. We have used here the definition given by Rippon and Stallard in [40] as follows.

Definition 2.1.1 (Fast escaping set). For a TEF $f$, the fast escaping set is a set of the form:

$$
A(f)=\left\{z: \exists L \in \mathbb{N} \text { such that }\left|f^{n+L}(z)\right| \geq M^{n}(R, f) \quad \forall n \in \mathbb{N}\right\}
$$

where $M(r, f)=\max _{|z|=r}|f(z)|, r>0$ and $M^{n}(r, f)$ denotes iteration of $M(r, f)$ with respect to $r$, and $R>0$ can be taken any value such that $M(r, f)>r$ for $r \geq R$.

The set $A(f)$ has many strong properties that can be used in the study of $I(f)$ and $J(f)$. Different properties of $A(f)$ and even different definitions of $A(f)$ were found in $[11,13,37,40]$. Significant progress in Eremenko's conjecture has been made possible by studying properties and structure of fast escaping set $A(f)$. The fundamental properties of this set are as follows.

Theorem 2.1.1. For a TEF $f$, the following statements are hold.

(1) $A(f)=A\left(f^{n}\right)$ for $n \geq 2$.

(2) $A(f) \neq \emptyset$.

(3) $A(f)$ is completely invariant.

(4) $A(f)$ is independent of $\mathrm{R}$.

(5) $J(f) \cap A(f) \neq \emptyset$.

(6) $J(f)=\partial A(f)$.

(7) $A(f)$ has no bounded components. 
The proof of the statement (1) is given in [37], the statements (2), (3) and (4) are stated in [13] and proved in [37], statements (5) and (6) are proved in [13, 37] and statement (7) is proved in [40]. This result (7) is an important one that provides a partial answer to Eremenko conjecture which is obtained on the basis of certain subsets of $A(f)$ based on above definition 2.1.1.

Definition 2.1.2 (Level of Fast Escaping Set). Let $f$ be a TEF. Let $L \in \mathbb{Z}$ and $R>0$ be such that $M(r, f)>r$ for $r \geq R$. The $L^{\text {th }}$ level of $A(f)$ with respect to $R$ is the set

$$
A_{R}^{L}(f)=\left\{z:\left|f^{n}(z)\right| \geq M^{n+L}(R, f) \text { for } n \in \mathbb{N}, n+L \geq 0\right\}
$$

In particular

$$
\begin{gathered}
A_{R}(f)=A_{R}^{0}(f)=\left\{z:\left|f^{n}(z)\right| \geq M^{n}(R, f) \text { for } n \in \mathbb{N}\right\} \text { and } \\
A(f)=\bigcup_{n \geq 0} f^{-n}\left(A_{R}(f)\right)
\end{gathered}
$$

Note that each of the level of $A(f)$ is a closed set. Since

$$
M^{n+1}(R, f)>M^{n}(R, f) \quad \forall n \geq 0
$$

So we have

$$
A_{R}^{L}(f) \subset A_{R}^{L-1}(f) \quad \forall L \in \mathbb{N}
$$

Also

$$
A(f)=\bigcup_{L \in \mathbb{N}} A_{R}^{-L}(f) \text { and } A_{R}^{-L}(f) \subset A_{R}^{-(L+1)}(f), L \in \mathbb{N}
$$

The concept of level as defined in the definition 2.1.2 provides a new understanding of the structure of $A(f)$ as a countable union of closed sets. On the basis of this definition, Rippon and Stallard [40] have obtained the strongest result for general TEF in the direction of Eremenko's conjecture which is nothing other than the statement (7) of above theorem 2.1.1.

Theorem 2.1.2. Let $f$ be a TEF and $R>0$ be such that $M(r, f)>r$ for $r \geq R$. Then for each $L \in \mathbb{Z}$, each component of $A_{R}^{L}(f)$ is closed and unbounded. In particular, each component of $A(f)$ is unbounded.

The proof of this theorem 2.1.2 is given in [40]. Since $A(f) \subset I(f)$, so this theorem provides partial answer to the Eremenko's conjecture 2.1 that $I(f)$ has at least one unbounded component. If $U$ is a Fatou component such that $U \cap A(f) \neq \emptyset$, then by normality $U \subset A(f)$. We say that such a Fatou component $U$ is fast escaping Fatou component. The following theorem due to Rippon and Stallard [37] gives important properties of the fast escaping Fatou component that provides us a contrasting feature of $A(f)$

Theorem 2.1.3. Let $f$ be a TEF and $R>0$ be such that $M(r, f)>r$ for $r \geq R$ and let $L \in \mathbb{Z}$. If $U$ is a Fatou component that meet $A_{R}^{L}(f)$, then

(1) $\bar{U} \subset A_{R}^{L-1}(f)$

(2) If, in addition $U$ is simply connected then $\bar{U} \subset A_{R}^{L}(f)$. 
This Theorem 2.1.3 implies that Fatou component $U$ of $A(f)$ has boundary in $A(f)$ but we have already mentioned that this does not happen in $I(f)$. In this context, Sixsmith [54] raised the question: Is there a TEF that can have simply connected fast escaping Fatou components without having multiply connected Fatou components? His affirmative answer is as follows:

Theorem 2.1.4. [54] There is a TEF with simply connected fast escaping Fatou component and no multiply connected Fatou components.

The levels $A_{R}^{L}(f)$ of $A(f)$ are also useful for the establishment of relationship between $A(f)$ and $J(f)$. In [40], this relation is shown in the following theorem.

Theorem 2.1.5. Let $f$ be transcendental entire function. Let $R>0$ be such that $M(r, f)>$ $r$ for $r \geq R$ and $L \in \mathbb{Z}$. Then all components of $A_{R}^{L}(f) \cap J(f)$ are unbounded if and only if $f$ has no multiply connected Fatou components.

This theorem 2.1.5 is the main result that provides an alternative condition for the partial solution of the normal version of Eremenko's conjecture. It says if $f$ has no multiply connected Fatou component, then all components of $A(f) \cap J(f)$ are unbounded. On the basis of theorem 2.1.4, there is a TEF that have simply connected fast escaping Fatou components. So we conclude that there is TEF in which $A_{R}^{L}(f) \cap J(f)$ are unbounded and hence all components of $A(f) \cap J(f)$ are unbounded. Which is a strong partial answer to the Eremenko's conjecture 2.1.

In recent years, active research in the field of escaping set has been devoted mostly to see the structure that has number of strong dynamical properties as well as able to establish the connection between the conjecture of Baker and the conjecture of Eremenko. The new research in this direction has become possible by the introduction of infinite spider's web. The first example of functions for which $A(f)$ has this structure have been given in [37]. Many TEF $f$ for which $A_{R}(f)$ and hence $A(f)$ has this structure has been given in [40]. This new set structure is defined as follows:

Definition 2.1.3 (Spider's Web). A set $E$ is an (infinite) spider's web if $E$ is connected and there exists a sequence of bounded simply connected domains $G_{n}$ with $G_{n} \subset G_{n+1}$ for $n \in \mathbb{N}, \partial G_{n} \subset E$ for $n \in \mathbb{N}$ and $\bigcup_{n \in \mathbb{N}} G_{n}=\mathbb{C}$.

We begin with basic properties of spider's web structure which are useful in proving the theorems 2.1.7, 2.2.1, 2.2.2.

Theorem 2.1.6. Let $f$ be a transcendental entire function and let $R>0$ be such that $M(r, f)>r$ for $r \geq R$ and $L \in \mathbb{Z}$.

(1) If G is a bounded components of $A_{R}^{L}(f)^{C}$, then $\partial G \subset A_{R}^{L}(f)$ and $f^{n}$ is a proper map of $\mathrm{G}$ onto the bounded component of $A_{R}^{n+L}(f)^{C}$, for each $n \in \mathbb{N}$.

(2) If $A_{R}^{L}(f)^{C}$ has bounded component, then $A_{R}^{L}(f)$ is a spider's web and hence every component of $A_{R}^{L}(f)^{C}$ is bounded.

(3) $A_{R}(f)$ is a spiders web if and only if $A_{R}^{L}(f)$ is a spider's web. 
(4) For $R^{\prime}>R$, then $A_{R}(f)$ is a spider's web if and only if $A_{R^{\prime}}(f)$ is a spiders web.

(5) If $I(f), J(f), I(f) \cap J(f)$ contain spider's web, then each of set is a spider's web.

Note that if $I(f)$ is a spider's web then $I(f)$ is connected and unbounded and so Eremenko's conjecture holds. In [37], Rippon and Stallard have proved that $A_{R}(f), A(f)$ and $I(f)$ are spider's web for a TEF $f$ whenever $f$ has multiply connected Faou component. In $[38,39]$, there are many TEF $f$ of sufficiently small growth such that $f$ has no multiply connected Fatou components and $A_{R}(f)$ is a spider's web. Rippon and Stallard proved the following strong results in $[40,42]$

Theorem 2.1.7. Let $f$ be a transcendental entire function and let $R>0$ be such that $M(r, f)>r$ for $r \geq R$.

(1) If $A_{R}(f)^{C}$ has a bounded component, then each of $A_{R}(f), A(f)$ and $I(f)$ is a spider's web.

(2) If $A_{R}(f)$ is a spider's web, then $A(f)^{C}$ has uncountably many components each of which is compact.

(3) If $A_{R}(f)$ is a spider's web, then $A(f)^{C}$ has singleton periodic components which are dense in $J(f)$.

(4) If $A_{R}(f)$ is a spider's web and $f$ has no multiply connected Fatou component, then each of $A_{R}(f) \cap J(f), A(f) \cap J(f), I(f) \cap J(f)$ and $J(f)$ is a spider's web.

(5) The function $f$ has no unbounded Fatou component.

This theorem 2.1.7 is a good example which shows a number of strong dynamical properties of $A_{R}(f)$ in the sense that when $A_{R}(f)$ is a spider's web, then so are $A(f)$ and $I(f)$. When $I(f)$ is a spider's web, then $I(f)$ is connected and unbounded, it follows that Eremenko's conjecture holds whenever $A_{R}(f)$ is a spider's web. The part $(2)$ of this theorem 2.1.7 demonstrates the fact that spider's web structure of $A(f)$ is connected with uncountably many complimentary components, each of which is closed and bounded. This contrasts with the fact that all components of $A_{R}^{L}(f)^{C}$ are open. This is a good example that $A(f)$ has very intricate structure, if $A_{R}(f)$ is a spider's web. Further results about the intricate structure of $A(f)$ are obtained by Osborne [30]. Part (5) of this theorem 2.1.7 provides a connection between the existence of an $A_{R}(f)$ spider's web and conjecture of Baker (Baker's conjecture is that if the order of TEF $f$ is less than $\frac{1}{2}$, then $f$ has unbounded Fatou components and it is dealt nicely in [4] and[5] and survey of the advances of this conjecture is found in [22]). Note that in [43], it is shown that if $f$ is a TEF of order less than $\frac{1}{2}$ and with all zeros in the negative real axis, then all components of $F(f)$ are bounded. From the above theorem 2.1.7 and all examples given in [40], we conclude that either $A(f)$ or $I(f)$ is a spider's web if $A_{R}(f)$ is a spider's web. However, the following statements are remained open.

Open Problem: Can $A(f)$ be a spider's web when $A_{R}(f)$ is not spider's wed?

Open Problem: Can $I(f)$ be a spider's web when $A_{R}(f)$ is not a spider's web?

2.2. Attempt of Proving Strong Version of Eremenko's Conjecture. The dynamical study of transcendental entire function was initiated by Fatou in 1926. In his memoire 
[21], Fatou observed from the function $f(z)=e^{-z}+z+1$ that there are infinitely many curves $\gamma_{k}, k \in \mathbb{N}$ such that $z \in \gamma_{k}, f^{n}(z) \rightarrow \infty$ as $n \rightarrow \infty$. Fatou then posed the question: Is this always true in the case of all transcendental entire functions? Sixty years after the Fatou's original question, Eremenko's made a precise study of the escaping set of transcendental entire functions and he posed his conjecture (strong version) 2.2.

The first family of transcendental entire functions whose escaping set has been investigated was the family of exponential functions:

$$
E_{\lambda}=\lambda e^{z}, \quad \lambda \in \mathbb{C}
$$

It was shown in [50] that every escaping point of this function can be connected to infinity along a curve consisting of escaping points. In the same paper [50], they provided complete classification of such escaping points and they organized in the form of differentiable curves called rays which are diffeomorphic to open intervals together with endpoints (landing points) of the ray. In fact, the most significant results in the direction of Eremenko's conjecture (strong version) given by Rottenfusser, Ruckert, Rempe and Schleicher in[47]. First of all, they provided an example of TEF $f \in \mathcal{B}$ such that every path connected components of $J(f)$ is bounded, together with the fact that $F(f) \cap I(f)=\emptyset$. This provides the answer for the question of Eremenko's conjecture in special case.

The feature of spider's web (definition 2.1.3) has become very important instrument of checking intricate structure of the set $A(f)$. The intricate nature (structure) of $A(f)$, where $A_{R}(f)$ is a spider's web has been investigated by Osborne in [28]. The following theorem of Rippon and Stallard [40] provides certain nature of $A(f)$ and $I(f)$ that helps to prove strong version of Eremenko's conjecture:

Theorem 2.2.1. Let $f$ be a transcendental entire function. Let $R>0$ be such that $M(R, f)>r$ for $r \geq R$ and $A_{R}(f)$ be a spider's web:

(1) Each point in $I(f)$ belongs to the unbounded continuum in $I(f)$ on which all points escape to infinity uniformly.

(2) If $K$ is a component of $A(f)^{c}$, then either $K \cap I(f)=\emptyset$ or all points in $K$ escape to infinity uniformly.

Part(1) of the Theorem 2.2.1 answers the question raised by Rempe in [34]. The same thing also holds for many functions in the Eremenko-Lyubich class $\mathcal{B}$ which consists of transcendental entire functions whose set of singular values is bounded. Rempe [36] also recognized a TEF in the class $\mathcal{B}$ such that every path connected components of $J(f)$ is bounded for which theorem 2.2.1(1) does not hold. Together with this fact and similar fact shown by Eremenko and Lyubich in [16] that $I(f) \subset J(f)$ (in particular $A(f) \subset J(f)$ ) if $f \in \mathcal{B}$. In such a case, Eremenko conjecture 2.2 does not hold in general.

On the other hand, for many transcendental entire functions in the class $\mathcal{B}$, the escaping set consists of family of curves tends to $\infty$. This situation occurs if $f$ is expressed as the finite composition of functions of finite order that are belonged to the class $\mathcal{B}$. In [36], this statement is proved for large class of TEF $f$ and also in [45] for the class $\mathcal{H}$ of functions 
satisfying head-start condition. This is the main result in the direction of Eremenko's conjecture (strong version) which is stated as follows:

Theorem 2.2.2. Suppose that $f: \mathbb{C} \rightarrow \mathbb{C}$ can be written as the finite composition $f=$ $f_{1} \circ f_{2} \circ f_{3} \circ \cdots \circ f_{n}$, where each $f_{i},(i=1,2,3 \ldots, n)$ is of bounded type (that is, $f_{i} \in \mathcal{B}$ ) and finite order, then $I(f) \cup\{\infty\}$ is path connected.

We discussed some cases where the strong version of Eremenko's conjecture holds. There are some transcendental entire functions that disprove this conjecture. In [45], Rottenfusser constructed a function $F$ in logarithmic coordinate such that there is an escaping point which can not connected to $\infty$ by a curve consisting of escaping points. As stated in the following theorem, there are many entire functions which do not have $A_{R}(f)$ spider's web. In particular, if $f \in \mathcal{B}$, then $A_{R}(f)$ is not a spider's web.

Theorem 2.2.3. Let $f$ be transcendental function. Let $R>0$ be such that $M(r, f)>r$ for $r \geq R$ and let $A_{R}(f)$ be a spider's web. Then there is no path to $\infty$ on which $f$ is bounded and so,

(1) $f$ does not belongs to the class $\mathcal{B}$.

(2) $f$ has no exceptional points (that is, points with finite backward orbits).

We have seen that for a given TEF $f$, if $A_{R}(f)$ is a spider's web, then each of sets $A(f)$ and $I(f)$ is spider's web. For such a function, the sets $\left.A_{R}(f)\right), A(f)$ and $I(f)$ are connected and $f$ has no bounded components and so both Eremenko's and Baker's conjecture hold. With these strong dynamical properties, it is better to ask: Which function $f$ that gives $A_{R}(f)$ a structure of spider's web? Several classes of functions that gives $A_{R}(f)$ a structure of spider's web are derived using the idea of following theorem.

Theorem 2.2.4. [40] Let $f$ be transcendental function. Let $R>0$ be such that $M(r, f)>r$ for $r \geq R$. Then $A_{R}(f)$ is a spider's web if one of the following holds:

(1) $f$ has a multiply connected Fatou component.

(2) $f$ has very small growth.

(3) $f$ has order less than $\frac{1}{2}$ and regular growth.

(4) $f$ has finite order, Fabry gaps and regular growth.

(5) $f$ has a sufficiently strong version of pits effects and has regular growth.

Parts (1) - (3) of this theorem 2.2.4 are given in [38] and [39], and the class of functions that are belong to $(4),(5)$ are defined and described in $[40,54]$. This theorem 2.2.4 is important one for determining a function $f$ for which $A_{R}(f)$ is a spider's web. Next, we give a criterion which allows us to construct many more functions if a function $f$ is known which gives $A_{R}(f)$ a structure of spider's web.

Theorem 2.2.5. Let $f$ be a TEF. Let $R>0$ be such that $M(r, f)>r$ for $r \geq R$. Then for $n \in \mathbb{N}, A_{R}\left(f^{n}\right)$ is a spider's web if and only if $A_{R}(f)$ is a spider's web. 


\section{Classes of Functions giving the Structure of Spider's Web}

In this section we try to elaborate the idea for the classes of functions given in theorem 2.2.4. This theorem tells us there are large classes of functions that give $A_{R}(f)$, a structure of escaping set whenever $M(r, f)>r$ for $r \geq R>0$. Part (1) of this theorem is nothing other than the following result which is a corollary of theorem 2.1.7(1). This theorem is proved in [40].

Theorem 3.1. Let $f$ be transcendental function. Let $R>0$ be such that $M(r, f)>r$ for $r \geq R$. If $f$ is multiply connected Fatou component, then each of sets $A_{R}(f), A(f)$ and $I(f)$ is a spider's web.

The classes of functions given in the rest parts (2)-(5) of theorem 2.2.4 are obtained by using the following general results of Rippon and Stallard [40].

Theorem 3.2. Let $f$ be transcendental function. Let $R>0$ be such that $M(r, f)>r$ for $r \geq R$. Then $A_{R}(f)$ is a spider's web if and only if there exists a sequence of bounded simply connected domains $\left(G_{n}\right)_{n \geq 0}$ such that

(1) $\left\{z:|z|<M^{n}(R)\right\} \subset G_{n}$, for all $n \geq 0$

(2) $G_{n+1}$ is contained in the bounded component of $\mathbb{C} \backslash f\left(\partial G_{n}\right)$ for all $n \geq 0$.

This result is very abstract and general. The essence of this theorem 3.2 holds if domains $G_{n}$ are replaced by discs. So the following result is considered a corollary of this theorem 3.2 and this will be more applicable in order to construct examples.

Theorem 3.3. Let $f$ be transcendental function. Let $R>0$ be such that $M(r, f)>r$ for $r \geq R$. Then $A_{R}(f)$ is a spider's web if there exists a sequence $\left(\rho_{n}\right)$ such that $\rho_{n}>M^{n}(R)$ and $m\left(\rho_{n}\right) \geq \rho_{n+1}$, for all $n \geq 0$. Where $m\left(\rho_{n}\right)$ is a minimum modulus function with respect to $\rho$.

We refer [22] for more detailed survey of this problem. The more strong results on this problem are given in [23] and [39]. In these papers, it is shown that Baker conjecture holds for all functions of small growth that have no unbounded Fatou components whenever the condition of this theorem 3.3 is satisfied. It is also shown in [39] that the conditions of this theorem are satisfied if $f$ is a TEF and and there exists $n \geq 2$ and $r_{0}>0$ such that

$$
\log \log M(r, f)<\frac{\log r}{\log ^{n} r} \text { for } r>r_{0}
$$

where $\log ^{n} r$ denotes $n t h$ iteration of $\log$ arithm function $\log r$. A TEF $f$ that satisfies this condition is called a function of arbitrarily small growth.

The following theorem gives a general result for other classes of functions which are not discussed above. For such classes of functions, the conclusion of theorem 3.2 holds. So, the following theorem is also a corollary of theorem 3.2 and this will also be more applicable in order to construct concrete examples.

Theorem 3.4. Let $f$ be transcendental function. Let $R>0$ be such that $M(r, f)>r$ for $r \geq R$. Then $A_{R}(f)$ is a spider's web if for some $m>1$ 
(1) there exists $\rho \in\left(r, r^{n}\right)$ with $m(\rho) \geq M(r, f)$, for all $r \geq R_{0}>0$, and

(2) there exists a sequence $\left(r_{n}\right)$ such that $r_{n}>M^{n}(R, f)$ and $M\left(r_{n}, f\right) \geq r_{n+1}^{m}$, for $n \geq 0$.

Note that function $f$ that satisfies the part (2) of this theorem 3.4 is called the function of regular growth and the condition is known as regularity condition. Note that there are more stronger regularity conditions than the condition given in this theorem 3.4(2). They are $\psi$-regularity and log-regularity where log-regularity is more stronger than $\psi$-regularity. We refer $[53,54]$, for more detailed study of both regularity conditions. In fact, if $f$ has order less than $\frac{1}{2}$, then $f$ satisfies part (1) of this theorem 3.4 for all sufficiently large values of $m$. If function $f$ has finite order and positive lower order, then $f$ satisfies part (2) of this theorem 3.4. For more detailed study of the existence of both part of this theorem 3.4 we refer $[1,39,40,54]$.

Now we have arrived in the position of to be more specific. From theorem 3.4, [22], [40] and [54], we have arrived the following conclusions.

Theorem 3.5. (1) If $f$ is a TEF of finite order and positive lower order, then $f$ is log-regular.

(2) If $f$ is a TEF of order less than $\frac{1}{2}$ and positive lower order, then $A_{R}(f)$ is a spider's web, where $R>0$ is such that $M(r, f)>r$ for $r \geq R$.

How to to produce classes of functions that satisfy theorem 3.5(2) ? As suggested in [54], the following operator will be quite helpful

$$
T_{m, n}(f(z))=\frac{1}{n} \sum_{k=1}^{n} f\left(e^{\frac{2 \pi i k}{n}} z^{\frac{m}{n}}\right)
$$

for all $m, n \in \mathbb{N}$, and $f$ is entire function. We choose a consistent branch of the nth root for each term in the sum. Note that this operator has the following elementary properties:

$$
T_{1, m} \circ T_{1, n}=T_{1, n m} \text { and } T_{m, n}\left(f\left(z^{n}\right)\right)=f\left(z^{n}\right)
$$

. The most important Property of this operator appears in the order and lower order of a function as shown in the following results.

Theorem 3.6. If $f$ is a TEF of order $\rho(f)$, then $T_{m, n}(f)$ is well defined entire function (for all $m, n \in \mathbb{N}$ ) of order at most $\frac{m}{n} \rho(f)$.

Theorem 3.7. Let $f(z)=\sum_{p=0}^{\infty} a_{p} z^{p}$ be a TEF and let $m, n \in \mathbb{N}$.

(1) If $\lim \inf _{p \rightarrow \infty} \frac{P \log p}{\log \left|a_{p n}\right|^{-1}}>0$, then $T_{m, n}(f)$ has positive lower order.

(2) If $T_{m, n}(f)$ has positive lower order and $g(z)=\sum_{p=0}^{\infty} b_{p} z^{p}$ be a TEF with $\left|b_{p}\right| \geq\left|a_{p}\right|$ for sufficiently large $p$, then $T_{m, n}(g)$ has a positive lower order.

For the proof of these both theorems 3.6, 3.7, we refer [54]. Finally, we examine some explicit classes of functions $g$ for which $A_{R}(g)$ is a spider's web.

Example 3.1. Let $f(z)=e^{z}$ and $g=T_{m, n}(f(z))$, where $n>2 m$. Then $A_{R}(g)$ is a spider's web, where $R>0$ is such that $M(r, g)>r$ for $r \geq R$. 
Solution: Since $\rho(f)=1$ and satisfies inequality of theorem 3.7(1) for $n>1 . g$ has order less than $\frac{1}{2}$ by theorem 3.6 and positive lower order by 3.7(1). Thus by theorem 3.5(2), $A_{R}(g)$ is a spider's web, where $R>0$ is such that $M(r, f)>r$ for $r \geq R$.

Example 3.2. Let $f(z)=z e^{z^{2}}+e^{z}$ and $g=T_{m, n}(f(z))$, where $n>4 m$ and $n$ is odd. Then $A_{R}(g)$ is a spider's web, where $R>0$ is such that $M(r, g)>r$ for $r \geq R$.

Solution: Since $\rho(f)=1$ and satisfies inequality of theorem 3.7(1) for odd $n(n>1)$. By theorem 3.6, $g$ has order less than $\frac{1}{2}$ and positive lower order by $3.7(2)$. So by theorem $3.5(2), A_{R}(g)$ is a spider's web, where $R>0$ is such that $M(r, f)>r$ for $r \geq R$.

As a particular case of example 3.1, the following is a famous TEF $g$ that gives $A_{R}(g)$, a structure of spider's web.

Example 3.3. Let $f(z)=e^{z}$ and $g=T_{2,4}(f(z))$, where $n \geq 2 m$. Then $A_{R}(g)$ is a spider's web, where $R>0$ is such that $M(r, g)>r$ for $r \geq R$.

Solution: From example 3.1, $A_{R}(g)$ is a spider's web, where $R>0$ is such that $M(r, f)>r$ for $r \geq R$. In this case, $g(z)=T_{2,4}(f(z))=\sum_{n=0}^{\infty} \frac{z^{n}}{(4 n) !}=\frac{1}{2}\left(\cos z^{\frac{1}{4}}+\cosh z^{\frac{1}{4}}\right)$. This function has order $\frac{1}{4}$ and positive lower order. In particular, for the function $h(z)=2 g\left(z^{4}\right)=$ $\cos z+\cosh z$, for which $A_{R}(h)$ is a spider's web.

For further explicit examples of classes of functions indicated by the theorem 2.2.4 that give $A_{R}(f)$, a structure of spider's web, we refer [38, 40, 54].

At the end of this section, we introduce some open problems arising from very familiar classes of transcendental entire functions about the structure of escaping set.

Let $\omega_{n}^{k}=e^{\frac{2 \pi i k}{n}}$ be $n$th roots of unity for some $n \in \mathbb{N}$ with $k=1,2, \ldots, n$. Let

$$
E_{n}=\left\{f: f(z)=\sum_{k=1}^{n} a_{k} e^{\left(\omega_{n}^{k} z\right)}, a_{k} \neq 0 \text { for } k=1,2, \ldots n\right\}
$$

In particular,

$$
E_{1}=\left\{f: f(z)=\lambda e^{z}, \lambda \in \mathbb{C}\right\}
$$

is a well known exponential family and

$$
E_{2}=\left\{f: f(z)=\alpha e^{z}+\beta e^{-z}, \alpha \neq 0, \beta \neq 0, \text { and } \alpha, \beta \in \mathbb{C}\right\}
$$

is well known cosine family upto conjugacy. As we indicated in introduction section, $E_{1}$ and $E_{2}$ are most familiar classes of TEF $f$ in Eremenko-Lyubich class $\mathcal{B}$.

From example 3.3, we observed that function $g(z)=\cos z+\cosh z$ gives $A_{R}(g)$, a structure of spider's web. We easily deduce that $g \in E_{4}$. In [54], Sixsmith drew the following statement as a open problem.

Open Problem: Is is true that if $f \in E_{n}$, for $n \geq 3$, then $A_{R}(f)$ is a spider's web?

Note that all functions in $E_{n}$ are log-regular and so from [41] that all functions in $E_{n}$ do not have multiply connected Fatou components. Therefore, if this question is solved, then by theorem 2.1.7(4), each of $A_{R}(f) \cap J(f), A(f) \cap J(f), I(f) \cap J(f)$ and $J(f)$ is a spider's web. 
Acknowledgment: We are thankful to Prof. Andrei Tetenov, Novosibirsk State University, Russia for his thorough review as well as valuable comments and suggestions as a referee of this survey paper.

\section{REFERENCES}

[1] J. M. Anderson and A. Hinkkanen, Unbounded Domain of Normality, Proc. Amer. Math. Soc. 126 (1998), 3243-3252.

[2] I. N. Baker, The Iteration of Entire Transcendental Function and Solution to the Functional Equation $(f(z))=F(z)$, Math. Annalen, Bd. 129, 5 (1955) 174-180.

[3] I. N. Baker, An Entire Function which has Wandering Domains, J. Austral. Math. Soc. 22 (Series-A) (1976), 173-176.

[4] I. N. Baker, The Iteration of Polynomials and Transcendental Entire Functions, J. Austral. Math.Soc. Series- A, 30 (1981), 483- 495.

[5] I. N. Baker, Wandering Domains in the Iteration of Entire Function, Proc. Math. Soc. (3), 49 (1984), 563- 576 .

[6] I. N. Baker, Dynamics of slowly Growing Entire Functions, Bull. Aust. Math..Soc.63(2001),367-377.

[7] W. Bergweiler, Iteration of Meromerphic Functions. Bull. Amer. Math. Soc. (N.S.) 29 (2) (1993), 151188.

[8] W. Bergweiler, On the Set where Iteration of Entire Functions is Bounded, Proc.Amer. Math. Soc. 140 3 (2012), 847-853.

[9] W. Bergweiler,, D. Drasin and A. Fletcher, The Fast Escaping Set of Quasi-regular Mappings.

[10] W. Bergweiler,, and A. Eremenko, Entire Functions of Slow Growth whose Julia Set coincides with the Plane, Ergodic Theory, Dyan. Syst., 20(2000), 1577-1582.

[11] W. Bergweiler, P. J. Rippon and G. M. Stallard, Dynamics of Meromorphic Functions with Direct or Logarithmic Singularities.

[12] W. Bergweiler, A. Fletcher, J. Langley and T. Meyer, The Escaping Set of Quasi- Regular Mappings, Proc. Amer Math. Soc., 137 (2009), 641- 651.

[13] W. Bergweiler and A. Hinkkanen, On the Semi- Conjugation of Entire Functions, Math. Proc. Camb. Phil. Soc. 126 (1999) 565- 574.

[14] L. Carleson and T. W. Gamelin, Complex Dynamics, Springer-Verlag, New York, 1992.

[15] A. Eremenko, On the Iteration of Entire Functions, Dynam. Sys. and Ergod. Theory, Banach Center Publications, polish Scientific Publishers, 23 (1989), 339-345.

[16] A. Eremenko and M. Y. Lyubich, Dynamical Properties of Some Classes of Entire Functions, Ann. Inst. Fourier, Grenoble, 42(1992), 989-1020.

[17] A. Eremenko and M.Y. Lyubich, The Dynamics of Analytic Transformations, Leningrad Math. J. Vol. 1, No. 3, (1990), 563- 587.

[18] A. Eremenko and M.Y. Lyubich, Iteration of Entire Functions, Soviet math. Dokl. 30 (3) (1984), 592594.

[19] A. Eremenko and M.Y. Lyubich, Examples of Entire Functions with Pathological Dynamics, J. London Math. Soc. (2) 86 (1987), 458-468.

[20] P. Fatou, Sur l'iteration Les Functions Transcendentes Entieres, Acta Math.47 (1926), 337- 370.

[21] P. Fatou, Sur les Equations Fuctionelles, Bull. Soc. Math. France 47 (1919), 161-271.

[22] A. Hinkanen, Entire Functions with Bounded Fatou Components. In Transcendental Dynamics and Complex Analysis, Vol. 348, London Math. Soc., Lecture Notes Ser., Pages 178-216, Cambridge Univ. Press, 2008.

[23] A. Hinkanen and J. Miles, Growth Conditions for the Entire Functions with only Bounded Fatou Components, Journal d'Analyse Math., 108 (2009), 87-118.

[24] X. N. Hua and C. C. Yang, Dynamics of Transcendental Functions. (Asians Mathematical Series), Gorden and Breach Science Publishers, Hong Kong China (1998). 
[25] G. Julia, Surlesileration des Functions Rationelles, J. Math. Pures Appl. (2) 4 (1918), $47-245$.

[26] J. Milner, Dynamics in One Complex Variables (Third Edition), Annals of Mathematical Studies, 160, Princeton University Press, Princeton, NJ, (2006).

[27] S. Morosawa , Y. Nishikura , M. Taniguchi and T. Ueda, Holomorphic Dynamics, Cambridge Studies in Advanced Mathematics 66, Cambridge University Press (1999).

[28] J. W. Osborne, The Structure of Spider's web Fast Escaping Sets, Bul. London Math.Soc. 44 (3) (2012), 503-519.

[29] J. W. Osborne, Spider's Web and Locally Connected Julia Sets of Transcendental Entire Functions, Ergodic Theory and Dynamical System, 33 (4) (2013), 1146-1161.

[30] Osborne, J.W.: The Structure of Spider's Web Fast Escaping Set, arXiv: 1011.059v1 [math.DS], 1 Nov., 2010.

[31] J. W. Osborne, Connectedness Properties of the Set where iterates of Entire Functions are Bounded, Math. Proc. Cambridge Philos. Soc.(2013), 155 (3),391-410.

[32] J. W. Osborne and P. J. Rippon and G. M. Stallard, Connected Properties of the Set where the Iteration of the Entire Functions is Unbounded, arXiv: 1504.5611.v1 [math.DS], 21 April, 2015.

[33] J. W. Osborne and D. J. Sixsmith, On the Set where the Iteration of an Entire Functions is neither Escaping nor Bounded, arXiv: 1503.08077 v1, [math.DS], 27 March, 2015.

[34] L. Rempe, On a question of Eremenko Concerning the Escaping Component of Entire Functions, Bull. London, Math. Soc. 39 (2007), 661-666.

[35] L. Rempe, Topological Dynamics of Exponential Maps on their Escaping Sets, Ergodic Theory Dynamical System 2636 (2006), 1933-1975.

[36] Rempe, L.: Dynamics of Entire Maps, PhD Thesis, Christian- Albrechts- Universitat, (2003).

[37] P. J. Rippon and G. M. Stallard, On Question of Fatou and Eremenko, Proc. Amer. Math. Soc. 139 (2005), 1119-1126.

[38] P. J. Rippon and G. M. Stallard, Escaping Point of Entire Functions of Small growth, Math. Z., 261(3)(2009) 557-570.

[39] P. J. Rippon and G. M. Stallard, Functions with Small growth with no unbounded Fatou Components, Journal d'Analyse Math., 108(2009), 61-86.

[40] P. J. Rippon and G. M. Stallard, Fast Escaping Point of Entire Function, proc. London Math. Soc. 105 (2012), 787-820.

[41] P. J. Rippon and G. M. Stallard, Boundaries of Escaping Fatou Components, Proc. Amer. Math. Soc. Vol.-139, No.-8, (2011), 2807-2820.

[42] P. J. Rippon, and G. M. Stallard, A Sharp Growth Condition for a Fast Escaping Spider's Web.

[43] P. J. Rippon and G. M. Stallard, Baker Conjecture and Eremenko's conjecture for functions with negetive real zeros, J. Anal. Math, Res. Not., arXiv:1208.3371v1, 2013.

[44] P. J. Rippon and G. M. Stallard, Regularity and Fast Escaping Points of Entire Functions, International Mathematics Research Notices, IMRN 2014 (2014), 5203-5229.

[45] G. Rottesfusser, On the Dynamical Fine Structure of Transcendental Entire, PhD Thesis, International University of Bremen (IUB), Germany, 2005.

[46] G. Rottesfusser and D. Schleicher, Escaping points of Cosine Family, In Transcendental Dynamics and Complex Analysis, Volume 348 of London Maths Society, Lecture Note Series, Page 396-424, Cambridge Uni. Press, Cambridge, 2008.

[47] G. Rottenfusser, J. Ruchert, L. Rempe and D. Schleicher, Dynamics Rays of Bounded Type Entire Functions, Ann. of Math. 173 (2011), 77-115.

[48] D. Schleicher, Dynamics of Entire Functions, Lecture Note of the CIME Summer School, Cosenza, Italy, (2008).

[49] D. Schleicher, textitDynamics of Entire Functions, July 8, 2008.

[50] D. Schleicher and J. Zimmer, Escaping Points of Exponential Maps, J. London Math. Soc. (2) 67 (2003), 380-400. 
[51] D. J. Sixsmith, Entire Functions for which the Escaping Set is Spider's Web, arXiv; 1212.1303v1 [math.DS], 6 Dec., 2010

[52] D. J. Sixsmith, Fast Simply Connected Escaping Fatou Components, arXiv: 1201.1926v1 [math.DS], 9 Jan., 2012.

[53] D. J. Sixsmith, On the Fundamental Loops and Fast Escaping Set, arXiv: 1301:2676v1 [math. DS], 12 June, 2013.

[54] D. J. Sixsmith, Topics in transcendental Dynamics, PhD Thesis, The Open University, UK, (2013).

[55] D. J. Sixsmith, Maximally and Non-maximally Fast Escaping Points of Transcendental Entire Functions, Math. Proc. Camb. Phil. Soc., (2015), in Press. 